Vom ökologischen zum nachhaltigen Konsum

\section{Genese eines Politikfeldes}

Schon lange ist es für die Umweltpolitik selbstverständlich, dass ihre Adressaten die Verursacher von Umweltbelastungen sind. Dennoch sind die gesellschaftlichen Konsequenzen daraus noch nicht ins Bewusstsein gedrungen: Auch die privaten Haushalte - und damit jede Bürgerin und jeder Bürger - gehören zu den Zielgruppen der Umweltpolitik. Eine dem Konzept der Nachhaltigkeit verpflichtete Politik muss darauf zielen, ihr Verhalten zu beeinflussen. Die praktische Umsetzung einer entsprechenden Konsumpolitik hinkt dieser Erkenntnis allerdings noch hinterher.

I Von Helmut Hagemann n den letzten Jahren hat sich die Aufmerksamkeit verstärkt dem privaten Verbrauch zugewandt (1). Nach einhelliger Expertenmeinung lässt sich ein großer Teil - laut Umweltbundesamt (UBA) 30-40 Prozent - aller Umweltprobleme direkt oder indirekt auf das Konsumverhalten zurückführen(2). Mit der Zuwendung zur Sphäre der Konsumenten wurde nachvollzogen, was in der Praxis bereits seit einiger Zeit manifest war: Der private Konsum wurde als umweltpolitisch bedeutsam erkannt. Ansätze zu ökologischem Konsumverhalten lassen sich in Deutschland schon in den Wurzeln der Umwelt- und Sozialbewegungen der siebziger Jahre ausmachen. In den achtziger Jahren wurde eine Vielzahl an Ratgebern für ökologisches Konsumverhalten publiziert. Daneben starteten viele soziale und wirtschaftliche Initiativen und Maßnahmen in diesem Bereich (3).

Nachhaltiger Konsum geht über das hinaus, was zunächst als ökologischer Konsum begriffen wurde: Er ist zum einen Gegenstand der Umweltpolitik und zum anderen Handlungsfeld innerhalb weitergehender Aktivitäten, die auf eine nachhaltige Entwicklung im Sinne der Brundtland-Kommission, der UNCED-Konferenz von 1992 sowie der daraus entstandenen Prozesse abzielen.

\section{- Internationale Impulse}

Die aus der UNCED-Konferenz entstandene Agenda 21 hat im Kapitel Vier ,Veränderung der Konsumgewohnheiten“ die „Entwicklung einer nationalen Politik und nationaler Strategien, um eine Änderung nicht nachhaltiger Verbrauchsgewohnheiten herbeizuführen" als eine Aufgabe benannt (4).
Diese Arbeit ist seither in einer Reihe von internationalen Ereignissen konkretisiert worden:

1993 verabschiedete die Commission for Sustainable Development (CSD) ein Arbeitsprogramm, das die Auseinandersetzung mit Produktions- und Konsummustern und die Erforschung von Lebensstilen zu einem Hauptanliegen erklärte.

- In den folgenden Jahren konkretisierte die CSD die Zielperspektiven für nachhaltige Produktions- und Konsummuster und regte unter anderem die Erstellung eines Indikatorensystems und einen aktionsorientierten Ansatz an.

- Auf europäischer Ebene betonte der verbraucherpolitische Aktionsplan 1999-2001 der Europäischen Union (EU) die Verantwortung der Verbraucher für die Umwelt (5).

- Die CSD widmete sich auf ihrer Tagung im April 1999 intensiv dem Thema „Promotion of sustainable consumption".

\section{Ansätze in Deutschland}

In Deutschland hat das Thema eine ähnliche Karriere vollzogen. Das UBA ging 1997 in seiner Studie „Nachhaltiges Deutschland“ auf die Möglichkeiten der Förderung nachhaltiger Konsummuster ein (6). In der Aufklärungsarbeit des UBA ist nachhaltiger Konsum zu einem Schwerpunkt geworden (7). Eine prominente Bedeutung hatte das Thema zuvor schon in der von BUND und Misereor herausgegebenen Studie „Zukunftsfähiges Deutschland" erfahren, in der die Autoren eine Orientierung an neuen Konsumleitbildern empfahlen. Desweiteren erfolgte eine Reihe von Fachtagungen (8) und Forschungsarbeiten (9). Mit dem „Demonstrationsvorhaben zur Fundierung und Evaluierung nachhaltiger Konsummuster und Verhaltensstile“ gab das UBA 1997 ein Verbundprojekt in Auftrag, das Unterstützung für die Förderung nachhaltiger Konsummuster geben soll (10). Unter der deutschen EU-Ratspräsidentschaft 1999 wurde im Rahmen einer Initiative zur integrierten Produktpolitik nachhaltiger Konsum als ein wichtiges Praxisfeld anerkannt. Eine weitere Aufwertung geschah durch die Erklärung der Bundesregierung zur Klimakonferenz 1999, in der die Verantwortung der Haushalte betont wurde.

\section{- Politische Perspektiven}

Wenngleich nachhaltiger Konsum sich als Politikfeld erst konstituiert, so wird er doch punktuell bereits durch verschiedene Ressorts gefördert. Hier können die Beispiele des Blauen Engels, die Regulierung von Neubauten nach Energieeffizienzstandards, die Förderung der Nachfrage nach umweltverträglicheren Lebensmitteln, die Aufklärung von Verbrauchern über umweltverträglichen Konsum und die Ökosteuer angeführt werden. Das Spektrum der Instrumente reicht vom Ordnungsrecht über marktwirtschaftliche Anreize bis zur Verbraucherbildung. Dabei sind die Wirkungen teilweise beachtlich (zum Beispiel bei der Senkung des spezifischen Heizwärmeverbrauchs), teilweise nur undeutlich zu erkennen (umweltbewusste Ernährungsberatung), was durchaus auf ihre geringe Ausstattung zurückgeführt werden kann.

Als kennzeichnend für die bisherige Praxis zeigt sich, dass eine Beeinflussung von Konsumentenverhalten eher einen marginalen Stellenwert hatte und manche Erfolge den Charakter von Nebenwirkungen zeigten. Zugleich wurden bestimmte Erfolge (wie etwa verbesserte Energieeffizienz oder erhöhte Naturkostumsätze) durch gegenläufige Trends (wie etwa Wohnflächenzunahme oder weitere Industrialisierung der Ernährung) gefährdet. Zudem war die Praxis durch mangelnde Kohärenz innerhalb und zwischen den Ressorts und durch mangelnde Kommunikation zwischen Staat, Gesellschaft und Wirtschaft geprägt. Nachhaltiger Konsum fristete angesichts dieser Situation ein Nischendasein, das bis dato nur flankierende politische Begleitung erfuhr.

Konsumentenverhalten im Sinne nachhaltiger Entwicklung zu beeinflussen, stellt eine komplexe Herausforderung dar. Die Verbraucher - als Objekte und Subjekte zugleich - wirken auf der Nachfrageseite der Konsumprozesse, die in engem Zusammenhang zur Angebotsseite steht. Nachhaltige Konsumpolitik muss dementsprechend den Einflussbereich der Verbraucher - 
von der Bedürfnisformulierung über Ge- und Verbrauch bis zur Entsorgung - erreichen. Sie muss ressortübergreifend, koordiniert und kooperativ angelegt sein und - in einer Arbeitsteilung von Staat, Gesellschaft und Wirtschaft bisherige Instrumente fortschreiben und besser ausstatten. Dabei wird es von großer Bedeutung sein, über Vermittlerinstanzen Zugang zu den verschiedenen sozialen Milieus und ihren Handlungsmöglichkeiten und vielfältigen Motivstrukturen zwischen den Polen von Ethik und Erlebnis zu finden. Ergänzt wird dies nach wie vor durch die Aufgabe, auf den anderen Lebenszyklusstufen der Konsumgüter, von den Vorprodukten über die Fertigung bis zum Vertrieb, eine nachhaltige Entwicklung zu unterstützen.

\section{Anmerkungen}

(1) Vgl. z.B. Neitzel H./ Landmann, U./ Pohl, M.: Das Umweltverhalten der Verbraucher - Daten und Tendenzen. Berlin 1994 und

Bodenstein, G./ Spiller, A. /Elbers, H.: Strategische Konsumentscheidungen: Langfristige Weichenstellung für das Umwelthandeln. Duisburg 1997.

(2) Umweltbundesamt: Nachhaltiges Deutschland. Berlin

1997, S. 221. Andere Autoren führten alle anthropogen bewegten Stoffströme auf eine Verursachung durch Konsum zurück, vgl. z.B. Weskamp, C. (Hrsg.): Ökologischer Konsum. Berlin 1995, S.7.

(3) Vgl. Sibum, D. et al: Nachhaltige Konsummuster und postmaterielle Lebensstile. Schwerpunkt 1: Bestandsaufnahme von Initiativen und Aktionen. Gelsenkirchen 1996 (4) Bundesumweltministerium (BMU) (Hrsg.): Konferenz der Vereinten Nationen für Umwelt und Entwicklung im Juni 1992 in Rio de Janeiro - Dokumente. Agenda 21. Bonn 1992, S. $22 f f$.

(5) Kommission der Europäischen Gemeinschaften: Verbraucherpolitischer Aktionsplan 1999-2001; Mitteilung der Kommission, Brüssel, 1.12.1998, KOM (1998) 696 endg.

(6) Umweltbundesamt: Nachhaltiges Deutschland. Berlin 1997, S. $220 f f$.

(7) Vgl. das Handbuch für den umweltbewussten Haushalt, UBA (Hrsg.): „Umweltbewusst Leben“, Berlin 1999.

(8) Z.B. Veranstaltungen des Deutschen Hygienemuseums (Oktober 1999), der Evangelischen Akademie Tutzing (März 1997), der Universität Hohenheim (1997), der Verbraucher-Zentrale NRW (1996), von UNESCO/ISOE

(1996), des Umweltbundesamts (1996) oder des Ökoforums (1994).

(9) Vgl. u.a. BMU: Umweltbewusstsein in Deutschland 1998. Berlin 1998;

Schluchter, W./ Dahm, G.: Analyse der Bedingungen für die Transformation von Umweltbewusstsein in umweltschonendes Verhalten. Berlin 1997;

Brand, K.W./ Poferl, A./ Schilling, K.: Determinanten des Umweltbewusstseins im Alltag, München 1996

sowie den Schwerpunkt Nachhaltiger Konsum der Ausgabe 3-4/96 von Ökologisches Wirtschaften.

(10) Das IÖW untersucht dabei unter Leitung des Autors politische Instrumente und Strategien, das ISOE erforscht ökologische Konsumententypen, das Wuppertal Institu† ökologische Prioritäten und Indikatoren und das IfAV sucht die Verständigung zwischen den relevanten Akteuren zu fördern (vgl. Meldung S. 26).

\section{Der Autor}

Dr. Helmut Hagemann, Sachverständiger für ökologischen Konsum und nachhaltige Entwicklung, leitete das Forschungsfeld Ökologischer Konsum des IÖW.

Kontakt: Germaniastr. 152B, 12099 Berlin, Tel. 030/ 76008701,

E-mail: Hagemann.Helmut@t-online.de

\section{LITERATUR ZUM SCHWERPUNKT}

Bodenstein, G./ Spiller, A./ Elbers, H.: Strategische Konsumentscheidungen: Langfristige Weichenstellung für das Umwelthandeln - Ergebnisse einer empirischen Studie. Duisburg 1997.

Brand, K.W./ Poferl, A./ Schilling, K.: Determinanten des Umweltbewusstseins im Alltag. Forschungsvorhaben des UBA. München 1996.

Brand, K.-W. (Hrsg.): Nachhaltige Entwicklung. Eine Herausforderung an die Soziologie. Opladen 1997.

\section{BUND / Misereor (Hrsg.): Zukunftsfähiges}

Deutschland. Ein Beitrag zu einer global nachhaltigen Entwicklung. Basel, Boston, Berlin 1996.

Dieckmann, A./ Preisendörfer, P.: Persönliches Umweltverhalten: Diskrepanzen zwischen Anspruch und Wirklichkeit. In: Kölner Zeitschrift für Soziologie und Sozialpsychologie, Jg. 44 (1992), Heft 2, S. 226-251.

Dierkes, M./ Fietkau, H.J.: Umweltbewusstsein Umweltverhalten. Materialien zur Umwelfforschung. Stuttgart 1988.

Enquete-Kommission „Schutz des Menschen und der Umwelt" (Hrsg.): Konzept Nachhaltigkeit. Fundamente für die Gesellschaft von morgen. Bonn 1997.
Gräbe, S. (Hrsg.): Private Haushalte im Spannungsfeld von Ökologie und Ökonomie. Frankfurt/M. New York 1993.

Hagemann, Helmut: Umweltverhalten zwischen Arbeit, Einkommen und Lebensstil. Konsumentenverhalten im Spannungsfeld von subjektiven Orientierungsmustern und Arbeitszeit- und Einkommensveränderungen. IÖW-Schriftenreihe 131/98, Berlin 1998 .

Neitzel, H./ Landmann, U./ Pohl, M.: Das Umweltverhalten der Verbraucher - Daten und Tendenzen. Empirische Grundlagen zur Konzipierung von "Sustainable Consumption Patterns" - Elemente einer "Ökobilanz Haushalte". Berlin 1994.

Reusswig, F.: Lebensstile und Ökologie. Gesellschaftliche Pluralisierung und alltagsökologische Entwicklung unter besonderer Berücksichtigung des Energiebereichs. ISOE, Frankfurt a.M. 1994.

Scherhorn, G. et al.: Wege zu nachhaltigen Konsummustern: Überblick über den Stand der Forschung und vorrangige Forschungsthemen. Ergebnisbericht über den Expertenworkshop „Wege zu nachhaltigen Konsummustern" des BMBF. Marburg 1997.
Schluchter, W./ Dahm, G.: Analyse der Bedingungen für die Transformation von Umweltbewusstsein in umweltschonendes Verhalten. UBA, Berlin 1997.

Schultz, I./ Weller, I.: Bestandsaufnahme der Ergebnisse der einschlägigen sozialwissenschaftlichen Forschung zu den Themenkreisen Umweltbewusstsein und -verhalten, Wertewandel, neve Lebensstile und neve Wohlstandsmodelle. In: Umweltbundesamt: Nachhaltige Konsummuster und postmaterielle Lebensstile. Vorstudien. Berlin 1997.

Umweltbundesamt (Hrsg.): Trendsetter - Schritte zum nachhaltigen Konsumverhalten am Beispiel der privaten Haushalte. Initativen - Projekte - Rahmenbedingungen. Berlin 1997.

Umweltbundesamt: Nachhaltiges Deutschland. Wege zu einer dauerhaft-umweltgerechten Entwicklung. Berlin 1997.

Weskamp, Cornelia (Hrsg.): Ökologischer Konsum. Ansätze und Leitbilder nachhaltig ökologischer und sozialverträglicher Lebensweisen. Berlin 1995. 
(c) 20I0 Authors; licensee IÖW and oekom verlag. This is an article distributed under the terms of the Creative Commons Attribution Non-Commercial No Derivates License (http://creativecommons.org/licenses/by-nc-nd/3.o/), which permits unrestricted use, distribution, and reproduction in any medium, provided the original work is properly cited. 